the volume by omitting altogether those sections dealing with endocrine and "trophic" disorders. In this way space has been found for a great deal of new material. The text-book can now be considered "up-to-date" and it includes references to electro-encephalography, the neuro-avitaminoses, and the neutropic virus affections. Dr. Russell Brain has also added an important and very readable chapter on the psychological manifestations of organic nervous disease.

This is a very excellent text-book of neurology, and indeed for the graduate studying for higher degrees, as well as for the brighter medical student, this book can confidently be recommended as the most satisfactory investment at present available.

\section{AN INTRODUCTION TO BIOCHEMISTRY}

\section{(Second Edition).}

By W. R. Fearon, M.A., Sc.D., M.B., F.I.C. William Heinemann Medical Books, Ltd. London. 1940. Price 17s. 6d.

Professor Fearon as he says in his preface, has approached his subject from the unusual path of inorganic biochemistry. In pursuance of this aim Part I of the book contains an extensive and informative chapter devoted to a consideration of not only the more common but also of those rarer elements concerned in the phenomenon of life; incidentally this chapter contains material from the Transactions of the U.S.S.R. Academy of Science not hitherto available in the English language. The remainder of Part $I$ is concerned with inorganic compounds, and such principles of physical chemistry as the Ionic Theory, Donnan Equilibrium, and Colloids without a thorough understanding of which the nature of the fundamental differences between the body and the test tube cannot be grasped.

An admirable compromise has been struck between facts and theories, and whilst the book is altogether exceptional in the quantity of very recent work incorporated, the author has kept in mind the important fact that the book is being written not for biochemists, but for less knowledgeable persons who require a simplified and more dogmatic presentation of the subject.

It is a pity that Professor Fearon has not mentioned in his chapters on Hormones that while the traditional view is that the parathyroid hormone controls the blood calcium level, a growing body of opinion considers that this effect is secondary, and that the primary effect of the hormone is upon the blood inorganic phosphate level; of particular interest in this connection is the recent paper of Helfet (Brit. Jr. of Surg., April, 1940) in which the implications of this latter theory are applied clinically with promising results.

Perhaps a rather greater degree of familiarity with the fundamentals of organic chemistry has been assumed for readers of this book than is usually the case with this type of publication, though certainly no excessive assumptions have been made on these grounds. The space saved by the omission of this elementary organic chemistry has been employed in the expansion of the chapters on such subjects of rapidly increasing importance as Tissue Respiration.

The average medical student will probably find this book a little long and a trifle too detailed for his needs as it contains considerably more material than that usually demanded for examination purposes, at the same time the reader of this book will acquire quite a fair knowledge of pure physiology.

While not a practical manual this book contains instructions for practical work to illustrate the text, this is of course admirable as a means of bringing practice and theory into proper proportion. A short appendix has been included, which lists the composition and uses of the chief biochemical reagents.

The author's style is both clear and concise, and care has been taken to prune the text of ambiguous or misleading statements in the avoidance of which pitfalls the author has been uniformly successful.

This is a book which if not one of the most simple is most certainly one of the best introductions to the subject yet written, and is to be recommended to all who wish to obtain an insight into the trend of modern biochemistry.

\section{ELEMENTARY PATHOLOGICAL HISTOLOGY.}

By W. G. Barnard, F.R.C.P. (2nd Edition). Crown 4to. H. K. Lewis \& Co., Ltd. 1940. Price 10s. 0d. net.

This book, which is of the nature of an atlas of morbid histology, contains 181 illustrations of which 8 are in colour. In view of the expense of producing these, the book is an object lesson on what can be done for the modest sum of ten shillings.

The author has had the courage to do what the author of this review has always longed to be able to do-to produce a text-book on morbid histology, illustrated largely by photomicrographs. In other contemporary works on this subject (notably that by the late Prof. Donaldson), the authors have sought to make things easier for the student by utilising only careful drawings, mostly imitations of photomicrographs, at the production of which the late Mr. Richard Muir so greatly excelled. While these are admirably lucid, they have the disadvantage that they do not really resemble what the student sees through the microscope. The illustrations in this book consist largely (though not entirely) of excellent photomicrographs which, though in monochrome, really do show the picture seen through the microscope.

The author is to be congratulated on these photomicrographs, which are of a high order of excellence. Almost anyone can get a good photomicrograph of such tissues as thyroid, but it takes real skill to produce clear crisp pictures of, for example, the edge of a gumma. The flatness of field of the larger photomicrographs is surprising to those who know how difficult it is to attain. 
If a criticism of these illustrations may be permitted, it may perhaps be said that the author has, if anything, been too lavish with them over some aspects of his subject while remaining deficient elsewhere. There are, for example, no less than twelve illustrations of pericarditis alone, and some of the space so employed might well have been devoted to sections of tissues in cases of leukæmia (apparently not mentioned in the book). The inclusion in the book of such a rarity as a Wilm's tumour of the kidney seems to suggest that the omission was not merely on the grounds of the rarity of leukæmia. It is noteworthy, however, that no blood conditions receive attention, which is, perhaps, excusable in a book that only purports to show the student the approaches to morbid histology. It seems a pity, however, that the author could not, while he was about it, accompany the student a little further along the path he has so excellently marked out.

\section{ACUTE INFECTIOUS DISEASES.}

By J. D. Rolleston and G. W. Ronaldson. 3rd edition. Pp. 477. Wm. Heinemann (Medical Books) Ltd. 1940. Price 17s 6d. net.

This is a book for the post-graduate rather than for the student. It abounds in specialised knowledge and has distinct literary quality. In the third edition the full historical and clinical descriptions of the infectious diseases treated in fever hospitals are retained and there is valuable additional information on methods of diagnosis, prophylaxis and treatment which have come into use during the past ten years. This greatly improves the general balance of this textbook and enhances its practical value. The authors have limited their writing to diseases which they have studied in the wards and in the literature of several languages for a lifetime. The references are legion. It is safe to say that no work of any importance bearing on the subject has been omitted. Indeed some of the references to earlier work might with advantage have been dropped in this edition.

One also feels occasionally that work of minor importance receives undue emphasis doubtless in a laudable endeavour to present as complete a view as possible. On treatment some minor criticisms may be offered. It is doubtful if the drip method of sero-therapy is necessary to-day, in view of the recent introduction of "digest" serum with its high concentration and low production of reactions. In laryngeal diphtheria it is stated that " aspiration has not secured many adherents in this country." Many who use this method regularly with success will disagree. The chapters on diphtheria, scarlet fever, measles, smallpox, typhoid and typhus fevers are probably the best presentations of the subject in British literature and will remain the standard work of reference in this country.

The book concludes with a valuable chapter on isolation methods illustrated by plans of various types of isolation wards.

\section{PHYSICAL DIAGNOSIS. (Elmer \& Rose)}

Revised by Harry Walker, M.D., F.A.C.P. 8th edition. Pp. 765, with 295 illustrations. Henry Kimpton. London, 1940. Price 45s. 0d. net.

The appearance of yet another edition of this book is in itself a testimony to the popularity and general usefulness of the work. In revising the text Dr. Walker has made some alterations in the order of the chapters with the idea of presenting the details of physical examination more in relation to the sequence usually observed in practice in regard to the examination of patients. The older arrangement, adopted in the 7th edition, appeared to us to be in some ways preferable, and on the whole simpler to follow, though doubtless the alteration has been dictated by the author's experience of the needs of his students and of readers in general.

In the preface Dr. Walker refers to the limitations of physical diagnosis which have been demonstrated by modern and improved diagnostic methods and to the worthlessness of many physical signs on which formerly so much reliance was often placed. In spite of this, however, he has chosen not to omit these in his description, maintaining that only thus is it possible fairly to assess the relative values of the different objective signs of disease, the importance of such assessment being succinctly emphasized in the short introduction given in the first chapter.

In view of the great advances that have been made in the last quarter of a century in our methods of diagnosis and of the clearer approach to the problems of disease which has resulted therefrom, it may appear to some that much of the detail which has been set down in this book is superfluous and, from a practical point of view, confusing to the student. We are inclined to think that this is the case, but in making this observation we merely call attention to a disadvantage inherent in any work of this character, nor do we intend it in the least as an adverse criticism of this particular volume, which has the merit of completeness, and which shows evidence of no little care and thoroughness in its preparation. The methods by which the art of diagnosis is acquired vary considerably according to the trend of the individual mind. For those, and there are many, who assimilate their knowledge more easily by the aid of a systematic text-book this volume supplies a much needed and reliable source of information.

The chapter dealing with the examination of nervous diseases is, to our mind, less convincing than the rest of the book; it would have been impraved by the inclusion of more diagrams illustrative of the anatomy of the central nervous system. We regret the omission of a separate chapter on radiology in physical diagnosis, such as appeared in the 7 th edition. The additional description of the use of the bronchoscope is welcome, but might well have been vouchsafed more space in view of the great importance of this. instrument in modern diagnostic work. 